\title{
ON GLOBALLY GENERATED VECTOR BUNDLES ON PROJECTIVE SPACES II
}

\author{
JOSÉ CARLOS SIERRA* AND LUCA UGAGLIA
}

\begin{abstract}
Extending the main result of [12, we classify globally generated vector bundles on $\mathbb{P}^{n}$ with first Chern class equal to 3 .
\end{abstract}

\section{MAIN RESULT}

The main result of the paper is the following.

Theorem 1.1. Let $\mathcal{E}$ be a globally generated vector bundle of rank $k$ on $\mathbb{P}^{n}$. If $c_{1}(\mathcal{E})=3$ and $c_{2}(\mathcal{E}) \leq 4$ then one of the following holds:

(i) $c_{2}(\mathcal{E})=0$ and $\mathcal{E}=\mathcal{O}_{\mathbb{P}^{n}}(3)$;

(ii) $c_{2}(\mathcal{E})=2$ and $\mathcal{E}=\mathcal{O}_{\mathbb{P}^{n}}(2) \oplus \mathcal{O}_{\mathbb{P}^{n}}(1)$;

(iii) $c_{2}(\mathcal{E})=3$ and $\mathcal{E}=\mathcal{O}_{\mathbb{P}^{n}}(2) \oplus T_{\mathbb{P}^{n}}(-1)$;

(iv) $c_{2}(\mathcal{E})=3$ and $\mathcal{E}=\mathcal{O}_{\mathbb{P}^{n}}(1) \oplus \mathcal{O}_{\mathbb{P} n}(1) \oplus \mathcal{O}_{\mathbb{P} n}(1)$

(v) $c_{2}(\mathcal{E})=4$ and $\mathcal{E}=\mathcal{O}_{\mathbb{P}^{n}}(1) \oplus \mathcal{O}_{\mathbb{P}^{n}}(1) \oplus T_{\mathbb{P}^{n}}(-1)$;

(vi) $c_{2}(\mathcal{E})=4$ and $\mathcal{E}=\mathcal{O}_{\mathbb{P}^{3}}(1) \oplus \Omega_{\mathbb{P}^{3}}(2)$;

(vii) $c_{2}(\mathcal{E})=4$ and $\mathcal{E}=\Omega_{\mathbb{P}^{4}}(2)$;

(viii) $\mathcal{E}$ is given by an exact sequence $0 \rightarrow \mathcal{O}_{\mathbb{P} n}^{\oplus s} \rightarrow \mathcal{G} \oplus \mathcal{O}_{\mathbb{P} n}^{\oplus r} \rightarrow \mathcal{E} \rightarrow 0$, where $h^{0}\left(\mathcal{E}^{*}\right)=r, h^{1}\left(\mathcal{E}^{*}\right)=s$ and $\mathcal{G}$ is a bundle as above.

Theorem 1.1 immediately implies the following corollary.

Corollary 1.2. Let $\mathcal{E}$ be a globally generated vector bundle of rank $k$ on $\mathbb{P}^{n}$. If $c_{1}(\mathcal{E})=3$ then $\mathcal{E}$ is either as in Theorem 1.1, or one of the following holds:

(i) $c_{2}(\mathcal{E})=5$ and $\mathcal{E}=\Omega_{\mathbb{P}^{4}}^{2}(2)^{*}$;

(ii) $c_{2}(\mathcal{E})=5$ and $\mathcal{E}=T_{\mathbb{P}^{3}}(-1) \oplus \Omega_{\mathbb{P}^{3}}(2)$;

(iii) $c_{2}(\mathcal{E})=5$ and $\mathcal{E}=T_{\mathbb{P}^{n}}(-1) \oplus T_{\mathbb{P}^{n}}(-1) \oplus \mathcal{O}_{\mathbb{P}^{n}}(1)$;

(iv) $c_{2}(\mathcal{E})=6$ and $\mathcal{E}=T_{\mathbb{P}^{n}}(-1) \oplus T_{\mathbb{P}^{n}}(-1) \oplus T_{\mathbb{P}^{n}}(-1)$;

(v) $c_{2}(\mathcal{E})=6$ and $0 \rightarrow \mathcal{O}_{\mathbb{P}^{n}}(-2) \oplus \Omega_{\mathbb{P}^{n}}(1) \rightarrow \mathcal{O}_{\mathbb{P}^{n}}^{\oplus k+n+1} \rightarrow \mathcal{E} \rightarrow 0$;

(vi) $c_{2}(\mathcal{E})=7$ and $0 \rightarrow \mathcal{O}_{\mathbb{P} n}(-2) \oplus \mathcal{O}_{\mathbb{P} n}(-1) \rightarrow \mathcal{O}_{\mathbb{P} n}^{\oplus k+2} \rightarrow \mathcal{E} \rightarrow 0$;

(vii) $c_{2}(\mathcal{E})=9$ and $0 \rightarrow \mathcal{O}_{\mathbb{P}^{n}}(-3) \rightarrow \mathcal{O}_{\mathbb{P}^{n}}^{\oplus k+1} \rightarrow \mathcal{E} \rightarrow 0$;

(viii) $\mathcal{E}$ is given by $0 \rightarrow \mathcal{O}_{\mathbb{P}^{n}}^{\oplus s} \rightarrow \mathcal{G} \oplus \mathcal{O}_{\mathbb{P}^{n}}^{\oplus r} \rightarrow \mathcal{E} \rightarrow 0$, where $h^{0}\left(\mathcal{E}^{*}\right)=r, h^{1}\left(\mathcal{E}^{*}\right)=s$ and $\mathcal{G}$ is a bundle as above.

This note is a natural extension of [12. Therefore, we also want to thank the referee of that paper for his help.

Globally generated vector bundles $\mathcal{E}$ on $\mathbb{P}^{n}$ with $c_{1}(\mathcal{E})=3$ have also been studied independently, and using a different approach, in [10] and [1] (cf. Remark 2).

\section{Proof of Theorem 1.1}

We work over the field of complex numbers. Let $\mathcal{E}$ be a globally generated vector bundle on $\mathbb{P}^{n}$ of rank $k$, and let $\mathcal{E}^{*}$ denote its dual bundle. In view of the following result, we will assume throughout the paper that $h^{0}\left(\mathcal{E}^{*}\right)=h^{1}\left(\mathcal{E}^{*}\right)=0$.

* Research supported by the "Ramón y Cajal" contract RYC-2009-04999, the project MTM2009-06964 of MICINN and the ICMAT "Severo Ochoa" project SEV-2011-0087 of MINECO. 
Lemma 1 (First reduction). Let $\mathcal{E}$ be a globally generated vector bundle on $\mathbb{P}^{n}$. If $h^{0}\left(\mathcal{E}^{*}\right)=r$ and $h^{1}\left(\mathcal{E}^{*}\right)=s$, then there exists a globally generated vector bundle $\mathcal{G}$ such that $h^{0}\left(\mathcal{G}^{*}\right)=h^{1}\left(\mathcal{G}^{*}\right)=0$, and an exact sequence

$$
0 \rightarrow \mathcal{O}_{\mathbb{P} n}^{\oplus s} \rightarrow \mathcal{G} \oplus \mathcal{O}_{\mathbb{P} n}^{\oplus r} \rightarrow \mathcal{E} \rightarrow 0 .
$$

Proof. Just put together [12, Lemmas 3 and 4].

Let $c_{1}:=c_{1}(\mathcal{E})$ and $c_{2}:=c_{2}(\mathcal{E})$ denote the first and second Chern class of $\mathcal{E}$, respectively. We point out that $c_{1}^{2}-c_{2} \geq 0$ since $\mathcal{E}$ is globally generated. Furthermore, in order to classify globally generated vector bundles one can assume $c_{2} \leq \frac{c_{1}^{2}}{2}$ thanks to the following.

Lemma 2 (Second reduction). Let $\mathcal{E}$ be a globally generated vector bundle with Chern classes $c_{1}, c_{2}$. If $c_{2}>\frac{c_{1}^{2}}{2}$ then there exists a globally generated vector bundle $\mathcal{K}^{*}$, whose dual $\mathcal{K}$ is given by the exact sequence

$$
0 \rightarrow \mathcal{K} \rightarrow \mathcal{O}_{\mathbb{P}^{n}}^{\oplus h^{0}(\mathcal{E})} \rightarrow \mathcal{E} \rightarrow 0 .
$$

In particular, $c_{1}\left(\mathcal{K}^{*}\right)=c_{1}$ and $c_{2}\left(\mathcal{K}^{*}\right)=c_{1}^{2}-c_{2}<\frac{c_{1}^{2}}{2}$.

Proof. Consider the kernel $\mathcal{K}$ of the epimorphism $\mathcal{O}_{\mathbb{P}^{n}}^{\oplus h^{0}(\mathcal{E})} \rightarrow \mathcal{E} \rightarrow 0$.

Globally generated vector bundles with $c_{1} \leq 2$ were classified in [12]. From now on we concentrate on the case $c_{1}=3$. We start by considering the cases in which $\mathcal{E}$ admits a global section whose zero locus is a hypersurface in $\mathbb{P}^{n}$.

Proposition 2.1. If $h^{0}(\mathcal{E}(-3)) \neq 0$ then $\mathcal{E}=\mathcal{O}_{\mathbb{P}^{n}}(3)$. Moreover, if $h^{0}(\mathcal{E}(-3))=0$ then $h^{0}\left(\mathcal{E}_{K}(-3)\right)=0$ for every linear subspace $K \subset \mathbb{P}^{n}$ of dimension greater than 1 .

Proof. The first statement was shown in [12, Lemma 5]. On the other hand, if $h^{0}\left(\mathcal{E}_{K}(-3)\right) \neq 0$ then $\mathcal{E}_{K}=\mathcal{O}_{K}(3) \oplus \mathcal{O}_{K}^{\oplus k-1}$ by the first assertion and Lemma 1 Therefore $\mathcal{E}=\mathcal{O}_{\mathbb{P}^{n}}(3) \oplus \mathcal{O}_{\mathbb{P}^{n}}^{\oplus k-1}$ (see for instance [11, Ch. I, Theorem 2.3.2]), whence $h^{0}(\mathcal{E}(-3)) \neq 0$.

Proposition 2.2. Assume that $h^{0}(\mathcal{E}(-3))=0$.

(i) If $h^{0}(\mathcal{E}(-2)) \neq 0$ then either $\mathcal{E}=\mathcal{O}_{\mathbb{P}^{n}}(2) \oplus \mathcal{O}_{\mathbb{P}^{n}}(1)$, or $\mathcal{E}=\mathcal{O}_{\mathbb{P}^{n}}(2) \oplus$ $T_{\mathbb{P} n}(-1)$.

(ii) If $h^{0}(\mathcal{E}(-2))=0$ then $h^{0}\left(\mathcal{E}_{K}(-2)\right)=0$ for every linear subspace $K \subset \mathbb{P}^{n}$ of dimension greater than 1 .

Proof. To prove (i), we essentially argue as in [12, Proposition 3.2]. If $n=1$ the result is trivial, so we assume that $n \geq 2$. Let $s \in H^{0}(\mathcal{E}(-2))$ be a non-zero section. Consider the corresponding exact sequence of sheaves

$$
0 \rightarrow \mathcal{O}_{\mathbb{P} n} \rightarrow \mathcal{E}(-2) \rightarrow \mathcal{F} \rightarrow 0,
$$

and let $Z \subset \mathbb{P}^{n}$ be the zero locus of $s$. We claim that $Z$ is a finite scheme of length at most 1 . To get a contradiction, let $P, Q$ be two points (maybe infinitely close) where $s$ vanishes and let $L \subset \mathbb{P}^{n}$ be the line joining $P$ and $Q$. Restricting to $L$ and twisting, we get

$$
0 \rightarrow \mathcal{O}_{L}(2) \rightarrow \mathcal{E}_{L} \rightarrow \mathcal{F}_{L}(2) \rightarrow 0 .
$$

Since $\mathcal{E}$ is globally generated and $\mathcal{F}_{L}(2)$ is a quotient of $\mathcal{E}_{L}$, we deduce that $\mathcal{F}_{L}(2)$ is also globally generated. Furthermore

$$
3=c_{1}\left(\mathcal{E}_{L}\right)=c_{1}\left(\mathcal{O}_{L}(2)\right)+c_{1}\left(\mathcal{F}_{L}(2)\right)=2+c_{1}\left(\mathcal{F}_{L}(2)\right)
$$

and $P, Q \in Z$, so $s$ vanishes on $L$. Let $\mathbb{P}^{2} \subset \mathbb{P}^{n}$ be a general plane containing $L$. Then $s$ does not vanish identically on $\mathbb{P}^{2}$ as otherwise $s \in H^{0}(\mathcal{E}(-2))$ would be the 
zero section. Let $V \subset \mathbb{P}^{n}$ be the hypersurface of degree 2 where $s$ vanishes (considered as a section of $\mathcal{E})$. Then $s$ vanishes on $L$ and $V \cap \mathbb{P}^{2}$, whence $h^{0}\left(\mathcal{E}_{\mathbb{P}^{2}}(-3)\right) \neq 0$ contradicting Proposition 2.1] This proves the claim. Consider the restriction sequence

$$
0 \rightarrow \mathcal{O}_{H} \rightarrow \mathcal{E}_{H}(-2) \rightarrow \mathcal{F}_{H} \rightarrow 0
$$

to a hyperplane $H \subset \mathbb{P}^{n}$ not meeting $Z$. Then $\mathcal{F}_{H}$ is a vector bundle such that $\mathcal{F}_{H}(2)$ is globally generated and $c_{1}\left(\mathcal{F}_{H}(2)\right)=1$. Therefore $\mathcal{F}_{H}(2)$ is either $\mathcal{O}_{H}(1) \oplus$ $\mathcal{O}_{H}^{\oplus k-2}$ or $T_{H}(-1) \oplus \mathcal{O}_{H}^{\oplus k-n}$ by [12, Proposition 3.1]. As

$$
\mathcal{E}_{H}(-2) \in \operatorname{Ext}^{1}\left(\mathcal{F}_{H}, \mathcal{O}_{H}\right)=H^{n-2}\left(\mathcal{F}_{H}(-n)\right)=0,
$$

we deduce that $\mathcal{E}_{H}$ is either $\mathcal{O}_{H}(2) \oplus \mathcal{O}_{H}(1) \oplus \mathcal{O}_{H}^{\oplus k-2}$ or $\mathcal{O}_{H}(2) \oplus T_{H}(-1) \oplus \mathcal{O}_{H}^{\oplus k-n}$. We claim that $h^{n-1}(\mathcal{F}(-n-1))=0$. Assume that the claim is proved. Then

$$
\mathcal{E}(-2) \in \operatorname{Ext}^{1}\left(\mathcal{F}, \mathcal{O}_{\mathbb{P}^{n}}\right)=H^{n-1}(\mathcal{F}(-n-1))=0,
$$

whence $\mathcal{E}(-2)=\mathcal{O}_{\mathbb{P}^{n}} \oplus \mathcal{F}$ and $\mathcal{F}$ is a vector bundle such that $\mathcal{F}(2)$ is globally generated and $c_{1}(\mathcal{F}(2))=1$, so $\mathcal{E}$ is either $\mathcal{O}_{\mathbb{P}^{n}}(2) \oplus \mathcal{O}_{\mathbb{P}^{n}}(1)$ or $\mathcal{O}_{\mathbb{P}^{n}}(2) \oplus T_{\mathbb{P}^{n}}(-1)$ by [12, Proposition 3.1] and Lemma[1, Let us prove the claim. Consider the restriction sequence

$$
0 \rightarrow \mathcal{E}^{*} \rightarrow \mathcal{E}^{*}(1) \rightarrow \mathcal{E}_{H}^{*}(1) \rightarrow 0
$$

Since $h^{1}\left(\mathcal{E}^{*}\right)=0$ by assumption and $h^{1}\left(\mathcal{E}_{H}^{*}(1)\right)=0$, we get $h^{1}\left(\mathcal{E}^{*}(1)\right)=0$. Now consider the restriction sequence

$$
0 \rightarrow \mathcal{F}(-n-1) \rightarrow \mathcal{F}(-n) \rightarrow \mathcal{F}_{H}(-n) \rightarrow 0 .
$$

As $h^{n-2}\left(\mathcal{F}_{H}(-n)\right)=0$ and $h^{n-1}(\mathcal{F}(-n))=h^{n-1}(\mathcal{E}(-n-2))=h^{1}\left(\mathcal{E}^{*}(1)\right)=0$, we deduce that $h^{n-1}(\mathcal{F}(-n-1))=0$.

We now prove (ii). It suffices to show it for every hyperplane $H \subset \mathbb{P}^{n}$. If $h^{0}\left(\mathcal{E}_{H}(-2)\right) \neq 0$ for some hyperplane $H \subset \mathbb{P}^{n}$, we deduce from (i) and Lemma 1 that either $\mathcal{E}_{H}=\mathcal{O}_{H}(2) \oplus \mathcal{O}_{H}(1) \oplus \mathcal{O}_{H}^{\oplus k-2}$, or $\mathcal{E}_{H}$ fits in an exact sequence

$$
0 \rightarrow \mathcal{O}_{H}^{\oplus s} \rightarrow \mathcal{O}_{H}(2) \oplus T_{H}(-1) \oplus \mathcal{O}_{H}^{\oplus k+s-n} \rightarrow \mathcal{E}_{H} \rightarrow 0
$$

Assume first that $n \geq 4$. Then $h^{i}\left(\mathcal{E}_{H}(-j)\right)=0$ for $i=0,1$ and every $j \geq 3$. Consider the restriction sequence $0 \rightarrow \mathcal{E}(-j-1) \rightarrow \mathcal{E}(-j) \rightarrow \mathcal{E}_{H}(-j) \rightarrow 0$. We deduce from Serre's vanishing theorem that $h^{0}(\mathcal{E}(-3))=h^{1}(\mathcal{E}(-3))=0$, whence $h^{0}(\mathcal{E}(-2))=h^{0}\left(\mathcal{E}_{H}(-2)\right) \neq 0$. Now, assume that $n=3$. The Hirzebruch-RiemannRoch theorem yields $\chi(\mathcal{E})=\frac{1}{6}\left(c_{1}^{3}-3 c_{1} c_{2}+3 c_{3}\right)+c_{1}^{2}-2 c_{2}+\frac{22}{12} c_{1}+k$. Since $c_{1}=c_{2}=3$ we deduce that $\chi(\mathcal{E})=8+k+\frac{1}{2}\left(c_{3}+1\right)$. To get a contradiction, assume that $h^{0}(\mathcal{E}(-2))=0$. Then the restriction sequence gives $h^{0}(\mathcal{E}(-1)) \leq 3$ and $h^{0}(\mathcal{E}) \leq$ $9+k$. We deduce that $h^{3}(\mathcal{E})=h^{0}\left(\mathcal{E}^{*}(-4)\right)=0$ and $h^{2}(\mathcal{E})=h^{1}\left(\mathcal{E}^{*}(-4)\right)=0$ from Serre duality and the exact sequence $0 \rightarrow \mathcal{E}^{*}(-j-1) \rightarrow \mathcal{E}^{*}(-j) \rightarrow \mathcal{E}_{H}^{*}(-j) \rightarrow 0$. Hence $-h^{1}(\mathcal{E}) \geq\left(c_{3}-1\right) / 2$, that is, $c_{3}=1$ since $c_{3}$ is odd (see, for instance, 11, p. 113]). Therefore $\mathcal{E}=\mathcal{O}_{\mathbb{P}^{3}}(1)^{\oplus 3}$, and we get a contradiction.

Remark 1. We would like to thank Edoardo Ballico for pointing out the following gap in the proof of [12, Proposition 3.2]. The natural isomorphism between $H^{n-1}(\mathcal{F}(-n-1))$ and the dual of $H^{1}\left(\mathcal{F}^{*}\right)$ holds if the quotient $\mathcal{F}$ is a locally free sheaf, so we just have $\mathcal{E}(-1) \in \operatorname{Ext}^{1}\left(\mathcal{F}, \mathcal{O}_{\mathbb{P}^{n}}\right)=H^{n-1}(\mathcal{F}(-n-1))$. In order to show that $h^{n-1}(\mathcal{F}(-n-1))=0$, and hence $\mathcal{E}(-1)=\mathcal{F} \oplus \mathcal{O}_{\mathbb{P}^{n}}$, just note that $h^{n-1}(\mathcal{F}(-n))=h^{n-1}(\mathcal{E}(-n-1))=h^{1}\left(\mathcal{E}^{*}\right)=0$ and that $h^{n-2}\left(\mathcal{F}_{H}(-n)\right)=0(\mathrm{cf}$. Lemma 3 below).

The cases $h^{0}(\mathcal{E}(-3)) \neq 0$ and $h^{0}(\mathcal{E}(-2)) \neq 0$ were described in Propositions 2.1 and 2.2, respectively. Now we study in detail the case $h^{0}(\mathcal{E}(-1)) \neq 0$. 
Lemma 3. Let $s \in H^{0}(\mathcal{E}(-1))$ be a non-zero section, and let $0 \rightarrow \mathcal{O}_{\mathbb{P} n} \rightarrow \mathcal{E}(-1) \rightarrow$ $\mathcal{F} \rightarrow 0$ be the corresponding exact sequence of sheaves. If $h^{n-2}\left(\mathcal{F}_{H}(-n)\right)=0$ for some hyperplane $H \subset \mathbb{P}^{n}$ then $\mathcal{E}=\mathcal{O}_{\mathbb{P}^{n}}(1) \oplus \mathcal{F}(1)$. In particular, $\mathcal{F}(1)$ is a globally generated vector bundle with $c_{1}(\mathcal{F}(1))=2$.

Proof. We deduce that $h^{n-1}(\mathcal{F}(-n))=h^{n-1}(\mathcal{E}(-n-1))=h^{1}\left(\mathcal{E}^{*}\right)=0$ from the exact sequence $0 \rightarrow \mathcal{O}_{\mathbb{P} n}(-n) \rightarrow \mathcal{E}(-n-1) \rightarrow \mathcal{F}(-n) \rightarrow 0$, Serre duality, and the assumption that $h^{1}\left(\mathcal{E}^{*}\right)=0$ throughout the paper. Therefore, we get $h^{n-1}(\mathcal{F}(-n-$ $1))=0$ from the restriction sequence $0 \rightarrow \mathcal{F}(-n-1) \rightarrow \mathcal{F}(-n) \rightarrow \mathcal{F}_{H}(-n) \rightarrow 0$. As $\mathcal{E}(-1) \in \operatorname{Ext}^{1}\left(\mathcal{F}, \mathcal{O}_{\mathbb{P} n}\right)=H^{n-1}(\mathcal{F}(-n-1))=0$, we deduce that $\mathcal{E}(-1)=$ $\mathcal{O}_{\mathbb{P} n} \oplus \mathcal{F}$.

From now on, we also assume that $c_{2} \leq 4$ (cf. Lemma 2).

Proposition 2.3. If $h^{0}(\mathcal{E}(-2))=0$ and $h^{0}(\mathcal{E}(-1)) \neq 0$ then one of the following holds:

- $\mathcal{E}=\mathcal{O}_{\mathbb{P}^{n}}(1) \oplus \mathcal{O}_{\mathbb{P}^{n}}(1) \oplus \mathcal{O}_{\mathbb{P}^{n}}(1)$

- $\mathcal{E}=\mathcal{O}_{\mathbb{P}^{n}}(1) \oplus \mathcal{O}_{\mathbb{P}^{n}}(1) \oplus T_{\mathbb{P}^{n}}(-1)$

- $\mathcal{E}=\mathcal{O}_{\mathbb{P}^{3}}(1) \oplus \Omega_{\mathbb{P}^{3}}(2)$.

Proof. For $n=1$, the result is obvious, so we assume that $n \geq 2$. Let $s \in H^{0}(\mathcal{E}(-1))$ be a non-zero section, and consider the exact sequence of sheaves

$$
0 \rightarrow \mathcal{O}_{\mathbb{P} n} \rightarrow \mathcal{E}(-1) \rightarrow \mathcal{F} \rightarrow 0 .
$$

Let $Z \subset \mathbb{P}^{n}$ be the zero locus of $s$. We claim that $Z$ is a finite scheme of length at most 2. To get a contradiction, let $T \subset Z$ be a subscheme of length 3 and let $\Pi \subset \mathbb{P}^{n}$ be a plane containing $T$. Consider the restriction $\mathcal{E}_{\Pi}$ and the quotient

$$
0 \rightarrow \mathcal{O}_{\Pi}^{\oplus k-2} \rightarrow \mathcal{E}_{\Pi} \rightarrow \mathcal{Q} \rightarrow 0
$$

(cf. 11, Ch. I, Lemma 4.3.1]). Then $\mathcal{Q}$ is a globally generated vector bundle of rank $2, c_{1}(\mathcal{Q})=c_{1}\left(\mathcal{E}_{\Pi}\right)=3$ and $c_{2}(\mathcal{Q})=c_{2}\left(\mathcal{E}_{\Pi}\right) \leq 4$. The restriction to $\Pi$ of the non-zero section $s \in H^{0}(\mathcal{E}(-1))$ yields a non-zero section in $H^{0}\left(\mathcal{E}_{\Pi}(-1)\right)$ by Proposition 2.2(ii). Therefore, since $H^{0}\left(\mathcal{E}_{\Pi}(-1)\right) \cong H^{0}(\mathcal{Q}(-1))$, we get a non-zero section $\sigma \in H^{0}(\mathcal{Q}(-1))$ vanishing on $T \subset \Pi$. Since the zero locus of $\sigma$ is finite as otherwise $\sigma \in H^{0}(\mathcal{Q}(-2)) \cong H^{0}\left(\mathcal{E}_{\Pi}(-2)\right)=0$, we get $c_{2}(\mathcal{Q}(-1)) \geq 3$ contradicting the fact that

$$
c_{2}(\mathcal{Q}(-1))=(-1)^{2}-c_{1}(\mathcal{Q})+c_{2}(\mathcal{Q})=c_{2}(\mathcal{Q})-2 \leq 2,
$$

and hence proving the claim. Now consider the restriction

$$
0 \rightarrow \mathcal{O}_{H} \rightarrow \mathcal{E}_{H}(-1) \rightarrow \mathcal{F}_{H} \rightarrow 0
$$

to a hyperplane $H \subset \mathbb{P}^{n}$ such that $Z \cap H=\emptyset$. Then $\mathcal{F}_{H}(1)$ is a globally generated vector bundle, $c_{1}\left(\mathcal{F}_{H}(1)\right)=2$ and $c_{2}\left(\mathcal{F}_{H}(1)\right) \leq 2$. Therefore $\mathcal{F}_{H}(1)$ can be as in [12, Theorem 1.1], cases (i)-(iv). In case (i) we have $\mathcal{F}_{H}(1)=\mathcal{O}_{H}(2) \oplus \mathcal{O}_{H}^{\oplus k-2}$, so $h^{n-2}\left(\mathcal{F}_{H}(-n)\right)=0$ and hence $\mathcal{E}=\mathcal{O}_{\mathbb{P}^{n}}(1) \oplus \mathcal{O}_{\mathbb{P}^{n}}(2)$ by Lemma 3, giving a contradiction. In case (ii) we have $\mathcal{F}_{H}(1)=\mathcal{O}_{H}(1)^{\oplus 2} \oplus \mathcal{O}_{H}^{\oplus k-3}$, so $h^{n-2}\left(\mathcal{F}_{H}(-n)\right)=$ 0 and therefore $\mathcal{E}=\mathcal{O}_{\mathbb{P}^{n}}(1) \oplus \mathcal{O}_{\mathbb{P}^{n}}(1) \oplus \mathcal{O}_{\mathbb{P}^{n}}$ (1) by Lemma 3 In case (iv) we also have $h^{n-2}\left(\mathcal{F}_{H}(-n)\right)=0$. Therefore, by Lemma 3, $\mathcal{F}(1)$ is a globally generated vector bundle such that $\mathcal{F}_{H}(1)$ is either $\Omega_{\mathbb{P}^{3}}(2) \oplus \mathcal{O}_{\mathbb{P} 3}^{\oplus k-4}$ or $\mathcal{N}(1) \oplus \mathcal{O}_{\mathbb{P}^{3}}^{\oplus k-3}$, and we get a contradiction by [12, Theorem 1.1]. If $\mathcal{F}_{H}(1)$ is as in case (iii), we remark that $\mathcal{F}_{H}(1)$ is either $T_{H}(-1) \oplus \mathcal{O}_{H}(1) \oplus \mathcal{O}_{H}^{k-n-1}$ or $\mathcal{G} \oplus \mathcal{O}_{H}^{k-n}$, where $\mathcal{G}$ is a vector bundle of rank $n-1$ obtained as a quotient

$$
0 \rightarrow \mathcal{O}_{H} \rightarrow \mathcal{O}_{H}(1) \oplus T_{H}(-1) \rightarrow \mathcal{G} \rightarrow 0
$$

(cf. [12, Remark 3]). If $\mathcal{F}_{H}(1)=T_{H}(-1) \oplus \mathcal{O}_{H}(1) \oplus \mathcal{O}_{H}^{k-n-1}$ then $h^{n-2}\left(\mathcal{F}_{H}(-n)\right)=$ 0 , and hence $\mathcal{E}=\mathcal{O}_{\mathbb{P}^{n}}(1) \oplus \mathcal{F}(1)$ by Lemma 3, Therefore, $\mathcal{F}(1)$ is either $T(-1) \oplus$ 
$\mathcal{O}_{\mathbb{P}^{n}}(1)$ or $\Omega_{\mathbb{P}^{3}}(2)$ by [12, Theorem 1.1]. Let us see now that $\mathcal{F}_{H}(1)=\mathcal{G} \oplus \mathcal{O}_{H}^{k-n}$ yields a contradiction. Assume first that $n=3$. Then $h^{1}\left(\mathcal{F}_{H}(-3)\right)=h^{1}(\mathcal{G}(-4))=$ $h^{1}\left(\mathcal{G}^{*}(-2)\right)=0$, as $\mathcal{G}^{*}(-2) \cong \mathcal{G}(-4)$ since $\mathcal{G}$ is of rank 2 and $c_{1}(\mathcal{G}(-4))=-6$. Therefore, by Lemma $3 \mathcal{E}=\mathcal{O}_{\mathbb{P}^{3}}(1) \oplus \mathcal{F}(1)$, and hence $\mathcal{F}(1)=\mathcal{N}_{\mathbb{P}^{3}}(1) \oplus \mathcal{O}_{\mathbb{P}^{3}}^{\oplus k-3}$ by 12, Theorem 1.1]. This contradicts the assumption that $h^{1}\left(\mathcal{E}^{*}\right)=0$. Assume now that $n \geq 4$. To get a contradiction, we point out that $h^{1}\left(\mathcal{F}_{H}^{*}(-1)\right)=h^{1}\left(\mathcal{G}^{*}\right)=1$. Then it follows from the exact sequence

$$
0 \rightarrow \mathcal{F}_{H}^{*}(-1) \rightarrow \mathcal{E}_{H}^{*} \rightarrow \mathcal{O}_{H}(-1) \rightarrow 0
$$

that $h^{1}\left(\mathcal{E}_{H}^{*}\right)=1$. Hence the exact sequence

$$
0 \rightarrow \mathcal{E}^{*}(-1) \rightarrow \mathcal{E}^{*} \rightarrow \mathcal{E}_{H}^{*} \rightarrow 0
$$

yields $h^{2}\left(\mathcal{E}^{*}(-1)\right) \neq 0$, as we assume that $h^{1}\left(\mathcal{E}^{*}\right)=0$. Let us see that $h^{2}\left(\mathcal{E}^{*}(-2)\right)=$ 0 . Consider the exact sequence

$$
0 \rightarrow \mathcal{F}_{H}^{*}(-1-j) \rightarrow \mathcal{E}_{H}^{*}(-j) \rightarrow \mathcal{O}_{H}(-1-j) \rightarrow 0 .
$$

Then $h^{i}\left(\mathcal{E}_{H}^{*}(-j)\right)=h^{i}\left(\mathcal{F}_{H}^{*}(-1-j)\right)=h^{i}\left(\mathcal{G}^{*}(-j)\right)=0$ for $i \in\{1,2\}$ and every integer $j \geq 2$ (here we use $n \geq 4$ ). So we deduce from the exact sequence

$$
0 \rightarrow \mathcal{E}^{*}(-1-j) \rightarrow \mathcal{E}^{*}(-j) \rightarrow \mathcal{E}_{H}^{*}(-j) \rightarrow 0
$$

and Serre's vanishing theorem that $h^{2}\left(\mathcal{E}^{*}(-2)\right)=0$. Therefore, $h^{2}\left(\mathcal{E}^{*}(-2)\right)=$ 0 and $h^{2}\left(\mathcal{E}^{*}(-1)\right) \neq 0$ yields that $h^{2}\left(\mathcal{E}_{H}^{*}(-1)\right) \neq 0$, which is a contradiction as $h^{2}\left(\mathcal{E}_{H}^{*}(-1)\right)=h^{2}\left(\mathcal{F}_{H}^{*}(-2)\right)=h^{2}\left(\mathcal{G}^{*}(-1)\right)=0$.

Finally, we consider the case $h^{0}(\mathcal{E}(-1))=0$.

Corollary 2.4. Assume that $n \geq 3$. If $h^{0}(\mathcal{E}(-1))=0$ but $h^{0}\left(\mathcal{E}_{H}(-1)\right) \neq 0$ for some hyperplane $H \subset \mathbb{P}^{n}$, then $n=4$ and $\mathcal{E}_{\mathbb{P}^{3}}$ is either $\mathcal{O}_{\mathbb{P}^{3}}(1) \oplus \Omega_{\mathbb{P}^{3}}(2)$, or a quotient $0 \rightarrow \mathcal{O}_{\mathbb{P}^{3}} \rightarrow \mathcal{O}_{\mathbb{P}^{3}}(1) \oplus \Omega_{\mathbb{P}^{3}}(2) \rightarrow \mathcal{E}_{\mathbb{P}^{3}} \rightarrow 0$ of rank 3 .

Proof. Suppose first that $n \geq 4$. If $h^{0}\left(\mathcal{E}_{H}(-1)\right) \neq 0$ then it follows from Lemma 1 and Proposition 2.3 that $\mathcal{E}_{H}$ fits in an exact sequence $0 \rightarrow \mathcal{O}_{H}^{\oplus s} \rightarrow \mathcal{G} \oplus \mathcal{O}_{H}^{\oplus r} \rightarrow$ $\mathcal{E}_{H} \rightarrow 0$, where $r=h^{0}\left(\mathcal{E}_{H}^{*}\right), s=h^{1}\left(\mathcal{E}_{H}^{*}\right)$ and either

(i) $\mathcal{G}=\mathcal{O}_{H}(1)^{\oplus 3}$, or

(ii) $\mathcal{G}=\mathcal{O}_{H}(1)^{\oplus 2} \oplus T_{H}(-1)$, or

(iii) $\mathcal{G}=\mathcal{O}_{\mathbb{P}^{3}}(1) \oplus \Omega_{\mathbb{P}^{3}}(2)$.

In cases (i) and (ii) we get $h^{i}\left(\mathcal{E}_{H}(-j)\right)=h^{i}(\mathcal{G}(-j))=0$ for $i \in\{0,1\}$ and every integer $j \geq 2$ (here we use $n \geq 4$ ). Hence we deduce from the exact sequence

$$
0 \rightarrow \mathcal{E}(-j-1) \rightarrow \mathcal{E}(-j) \rightarrow \mathcal{E}_{H}(-j) \rightarrow 0
$$

and Serre's vanishing theorem that $h^{0}(\mathcal{E}(-2))=h^{1}(\mathcal{E}(-2))=0$. Therefore

$$
h^{0}(\mathcal{E}(-1))=h^{0}\left(\mathcal{E}_{H}(-1)\right) \neq 0,
$$

yielding a contradiction. Hence case (iii) holds and $n=4$. Furthermore, we claim that $h^{0}\left(\mathcal{E}_{H}^{*}\right)=0$. From the dual sequence $0 \rightarrow \mathcal{E}_{H}^{*} \rightarrow \mathcal{G}^{*} \oplus \mathcal{O}_{H}^{\oplus r} \rightarrow \mathcal{O}_{H}^{\oplus s} \rightarrow 0$ we deduce that $h^{i}\left(\mathcal{E}_{H}^{*}(-j)\right)=h^{i}\left(\mathcal{G}^{*}(-j)\right)=0$ for $i \in\{0,1\}$ and every integer $j \geq 1$. From the exact sequence

$$
0 \rightarrow \mathcal{E}^{*}(-1-j) \rightarrow \mathcal{E}^{*}(-j) \rightarrow \mathcal{E}_{H}^{*}(-j) \rightarrow 0
$$

and Serre's vanishing theorem we get $h^{0}\left(\mathcal{E}^{*}(-1)\right)=h^{1}\left(\mathcal{E}^{*}(-1)\right)=0$, and hence $h^{0}\left(\mathcal{E}_{H}^{*}\right)=h^{0}\left(\mathcal{E}^{*}\right)=0$. Therefore $\mathcal{E}_{H}$ is either $\mathcal{O}_{\mathbb{P}^{3}}(1) \oplus \Omega_{\mathbb{P}^{3}}(2)$, or a quotient $0 \rightarrow \mathcal{O}_{\mathbb{P}^{3}}^{\oplus s} \rightarrow \mathcal{O}_{\mathbb{P}^{3}}(1) \oplus \Omega_{\mathbb{P}^{3}}(2) \rightarrow \mathcal{E}_{H} \rightarrow 0$ where, in the latter, $s=1$ as $c_{3}\left(\mathcal{O}_{\mathbb{P}^{3}}(1) \oplus\right.$ $\left.\Omega_{\mathbb{P}^{3}}(2)\right) \neq 0$. 
Assume now that $n=3$. We argue as in Proposition 2.2. To get a contradiction, assume that $h^{0}(\mathcal{E}(-1))=0$ and $h^{0}\left(\mathcal{E}_{H}(-1)\right) \neq 0$. Then we deduce from Proposition 2.3 that $\mathcal{E}_{H}$ is given by an exact sequence

$$
0 \rightarrow \mathcal{O}_{H}^{\oplus s} \rightarrow \mathcal{O}_{H}(1)^{\oplus 2} \oplus T_{H}(-1) \oplus \mathcal{O}_{H}^{\oplus k+s-4} \rightarrow \mathcal{E}_{H} \rightarrow 0
$$

As $h^{0}(\mathcal{E}(-1))=0$, we deduce from the restriction sequence that $h^{0}(\mathcal{E}) \leq k+5$. We deduce that $h^{3}(\mathcal{E})=h^{0}\left(\mathcal{E}^{*}(-4)\right)=0$ and $h^{2}(\mathcal{E})=h^{1}\left(\mathcal{E}^{*}(-4)\right)=0$ from Serre duality and the exact sequence $0 \rightarrow \mathcal{E}^{*}(-1-j) \rightarrow \mathcal{E}^{*}(-j) \rightarrow \mathcal{E}_{H}^{*}(-j) \rightarrow 0$. By the Hirzebruch-Riemann-Roch theorem, we get $\chi(\mathcal{E})=\frac{1}{6}\left(c_{1}^{3}-3 c_{1} c_{2}+3 c_{3}\right)+c_{1}^{2}-2 c_{2}+$ $\frac{22}{12} c_{1}+k$, and hence $h^{0}(\mathcal{E})-h^{1}(\mathcal{E})=k+5+c_{3} / 2 \leq k+5-h^{1}(\mathcal{E})$; that is, $c_{3}=0$ giving a contradiction (see for instance [5, Theorem 1.1]).

Let us see that only the first case in Corollary 2.4 actually occurs.

Proposition 2.5. Assume that $h^{0}(\mathcal{E}(-1))=0$ but $h^{0}\left(\mathcal{E}_{H}(-1)\right) \neq 0$ for some hyperplane $H \subset \mathbb{P}^{4}$. Then $\mathcal{E} \cong \Omega_{\mathbb{P}^{4}}(2)$.

Proof. It follows from Corollary 2.4 that $\mathcal{E}_{\mathbb{P}^{3}}$ is either $\mathcal{O}_{\mathbb{P}^{3}}(1) \oplus \Omega_{\mathbb{P}^{3}}(2)$, or a quotient $0 \rightarrow \mathcal{O}_{\mathbb{P}^{3}} \rightarrow \mathcal{O}_{\mathbb{P}^{3}}(1) \oplus \Omega_{\mathbb{P}^{3}}(2) \rightarrow \mathcal{E}_{\mathbb{P}^{3}} \rightarrow 0$ of rank 3 .

If $\mathcal{E}_{H}=\mathcal{O}_{\mathbb{P}^{3}}(1) \oplus \Omega_{\mathbb{P}^{3}}(2)$ then we see from Serre's vanishing theorem and the restriction sequence

$$
0 \rightarrow \mathcal{E}(-j-1) \rightarrow \mathcal{E}(-j) \rightarrow \mathcal{E}_{H}(-j) \rightarrow 0
$$

that $h^{1}(\mathcal{E}(-2))=h^{1}\left(\mathcal{E}_{H}(-2)\right)=1$. Therefore we have a non-trivial extension

$$
0 \rightarrow \mathcal{O}_{\mathbb{P}^{4}} \rightarrow \mathcal{G} \rightarrow \mathcal{E}^{*}(2) \rightarrow 0
$$

We claim that $\mathcal{G}=\mathcal{O}_{\mathbb{P}^{4}}(1)^{\oplus 5}$. In view of [11, Ch. I, Theorem 2.3.2], it is enough to show that $\mathcal{G}_{H}=\mathcal{O}_{H}(1)^{\oplus 5}$. Let us see that $\mathcal{G}_{H}$ has no intermediate cohomology. From the exact sequence

$$
0 \rightarrow \mathcal{O}_{H} \rightarrow \mathcal{G}_{H} \rightarrow \mathcal{O}_{H}(1) \oplus T_{H} \rightarrow 0,
$$

we deduce that $h^{1}\left(\mathcal{G}_{H}(j)\right)=0$ for every integer $j$ and that $h^{2}\left(\mathcal{G}_{H}(j)\right)=0$ for every integer $j \neq-4$. For $j=-4$, we have $h^{2}\left(\mathcal{G}_{H}(-4)\right)=h^{1}\left(\mathcal{G}_{H}^{*}\right)$. It follows from the exact sequence

$$
0 \rightarrow \mathcal{O}_{H}(-1-j) \oplus \Omega_{H}(-j) \rightarrow \mathcal{G}_{H}^{*}(-j) \rightarrow \mathcal{O}_{H}(-j) \rightarrow 0
$$

that $h^{0}\left(\mathcal{G}_{H}^{*}(-j)\right)=h^{1}\left(\mathcal{G}_{H}^{*}(-j)\right)=h^{2}\left(\mathcal{G}_{H}^{*}(-j)\right)=0$ for every $j \geq 1$. Therefore Serre's vanishing theorem applied to the restriction sequence

$$
0 \rightarrow \mathcal{G}^{*}(-j-1) \rightarrow \mathcal{G}^{*}(-j) \rightarrow \mathcal{G}_{H}^{*}(-j) \rightarrow 0
$$

yields $h^{1}\left(\mathcal{G}^{*}(-1)\right)=h^{2}\left(\mathcal{G}^{*}(-1)\right)=0$, and hence $h^{1}\left(\mathcal{G}_{H}^{*}\right)=h^{1}\left(\mathcal{G}^{*}\right)=0$. Then Horrocks' theorem (see, for instance, [11, Ch. I, Theorem 2.3.1]) implies that $\mathcal{G}_{H}$ splits. Finally $c_{1}\left(\mathcal{G}_{H}\right)=5$ and $h^{0}\left(\mathcal{G}_{H}(-2)\right)=0$, so we get $\mathcal{G}_{H}=\mathcal{O}_{H}(1)^{\oplus 5}$. Then $\mathcal{E}=\Omega_{\mathbb{P}^{4}}(2)$.

Assume now that $\mathcal{E}_{H}$ is given by a quotient

$$
0 \rightarrow \mathcal{O}_{\mathbb{P}^{3}} \rightarrow \mathcal{O}_{\mathbb{P}^{3}}(1) \oplus \Omega_{\mathbb{P}^{3}}(2) \rightarrow \mathcal{E}_{\mathbb{P}^{3}} \rightarrow 0
$$

Then $c_{t}(\mathcal{E})=c_{t}\left(\mathcal{E}_{H}\right)=1+3 t+4 t^{2}+2 t^{3}$. Therefore, we get a contradiction by the Schwarzenberger condition $\left(S_{4}^{3}\right)$ [11, p.113] for $s=4$.

We can now prove Theorem 1.1

Proof of Theorem 1.1. We can assume that $h^{0}\left(\mathcal{E}^{*}\right)=h^{1}\left(\mathcal{E}^{*}\right)=0$ by Lemma 1 , otherwise we get case (viii). If $h^{0}(\mathcal{E}(-3)) \neq 0$, then we get case (i) by Proposition 2.1. If $h^{0}(\mathcal{E}(-3))=0$ but $h^{0}(\mathcal{E}(-2)) \neq 0$, then we get cases (ii) and (iii) by Proposition 2.2. If $h^{0}(\mathcal{E}(-2))=0$ but $h^{0}(\mathcal{E}(-1)) \neq 0$, then we get cases (iv), (v) and (vi) by Proposition 2.3. If $h^{0}(\mathcal{E}(-1))=0$ but $h^{0}\left(\mathcal{E}_{H}(-1)\right) \neq 0$ for some hyperplane $H \subset \mathbb{P}^{n}$, then we get case (vii), by Corollary 2.4 and Proposition 2.5. Furthermore, 
we claim that there is no vector bundle $\mathcal{E}$ on $\mathbb{P}^{5}$ such that $\mathcal{E}_{H}=\Omega_{\mathbb{P}^{4}}(2) \oplus \mathcal{O}_{\mathbb{P}^{4}}^{\oplus k-4}$. As $h^{i}\left(\mathcal{E}_{H}^{*}(-j)\right)=0$ for $i \in\{0,1\}$ and every integer $j \geq 1$, we deduce from Serre's vanishing theorem and the restriction sequence

$$
0 \rightarrow \mathcal{E}^{*}(-1-j) \rightarrow \mathcal{E}^{*}(-j) \rightarrow \mathcal{E}_{H}^{*}(-j) \rightarrow 0
$$

that $h^{i}\left(\mathcal{E}^{*}(-1)\right)=0$ for $i \in\{0,1\}$. Therefore $h^{0}\left(\mathcal{E}^{*}\right)=h^{0}\left(\mathcal{E}_{H}^{*}\right)=k-4$ and hence there exists a rank- 4 vector bundle $\mathcal{G}$ such that $\mathcal{E}=\mathcal{G} \oplus \mathcal{O}_{\mathbb{P}^{5}}^{\oplus k-4}$. Then $c_{t}(\mathcal{G})=$ $c_{t}\left(\mathcal{E}_{H}\right)=1+3 t+4 t^{2}+2 t^{3}+t^{4}$ and we get a contradiction by the Schwarzenberger condition $\left(S_{5}^{4}\right)$ [11, p.113] for $s=5$. This proves the claim. Finally, if $h^{0}(\mathcal{E}(-1))=0$ and $h^{0}\left(\mathcal{E}_{H}(-1)\right)=0$ for every hyperplane $H \subset \mathbb{P}^{n}$ then we get

$$
h^{0}(\mathcal{E}) \leq h^{0}\left(\mathcal{E}_{H}\right) \leq \cdots \leq h^{0}\left(\mathcal{E}_{\mathbb{P}^{2}}\right) \leq h^{0}\left(\mathcal{E}_{\mathbb{P}^{1}}\right)=k+3 .
$$

Let us see that this is impossible. Consider the exact sequence

$$
0 \rightarrow \mathcal{K} \rightarrow \mathcal{O}_{\mathbb{P}^{2}}^{\oplus h^{0}\left(\mathcal{E}_{\mathbb{P}^{2}}\right)} \rightarrow \mathcal{E}_{\mathbb{P}^{2}} \rightarrow 0
$$

where $\mathcal{K}$ is a vector bundle on $\mathbb{P}^{2}$ with $h^{0}(\mathcal{K})=h^{1}(\mathcal{K})=0, c_{1}(\mathcal{K})=-3$ and $c_{2}(\mathcal{K})=c_{2}\left(\mathcal{K}^{*}\right)=9-c_{2} \geq 5$. Then the Hirzebruch-Riemann-Roch theorem

$$
\chi(\mathcal{K})=\frac{1}{2}\left(c_{1}(\mathcal{K})^{2}-2 c_{2}(\mathcal{K})+3 c_{1}(\mathcal{K})\right)+r k(\mathcal{K})
$$

for vector bundles on $\mathbb{P}^{2}$ yields

$$
0 \leq h^{2}(\mathcal{K})=-c_{2}(\mathcal{K})+h^{0}\left(\mathcal{E}_{\mathbb{P}^{2}}\right)-k \leq-5+h^{0}\left(\mathcal{E}_{\mathbb{P}^{2}}\right)-k
$$

i.e. $h^{0}\left(\mathcal{E}_{\mathbb{P}^{2}}\right) \geq k+5$, so we get a contradiction.

As a consequence, we obtain the classification of globally generated vector bundles $\mathcal{E}$ on $\mathbb{P}^{n}$ with $c_{1}=3$ and no restriction on $c_{2}$.

Proof of Corollary 1.2. The proof follows from Theorem 1.1] and Lemmas 1 and 2.

Remark 2. Some well-known globally generated vector bundles seem to be hidden in Theorem 1.1(viii) (e.g., $T_{\mathbb{P}^{2}}$ ) and Corollary 1.2(viii) (e.g., the Tango bundle $\mathcal{T}$ given by the exact sequence $0 \rightarrow T_{\mathbb{P}^{4}}(-2) \rightarrow \mathcal{O}_{\mathbb{P}^{4}}^{\oplus 7} \rightarrow \mathcal{T} \rightarrow 0$; see, for instance, [11. Ch. I, §4]). They can be easily detected in our classification by means of [11, Ch. I, Lemmas 4.3.1 and 4.3.2]. In this context, we point out that the only globally generated vector bundle of rank $k$ on $\mathbb{P}^{n}$ with $c_{1}=3$ and $k<n$ which does not split is the Tango bundle $\mathcal{T}$ of rank 3 on $\mathbb{P}^{4}$, as one immediately deduces from Theorem 1.1 and Corollary 1.2 that $c_{n}(\mathcal{E})=0$ if and only if $\mathcal{E}=\Omega_{\mathbb{P}^{4}}^{2}(2)^{*} \cong \wedge^{2} T_{\mathbb{P}^{4}}(-2)$, giving the following diagram:

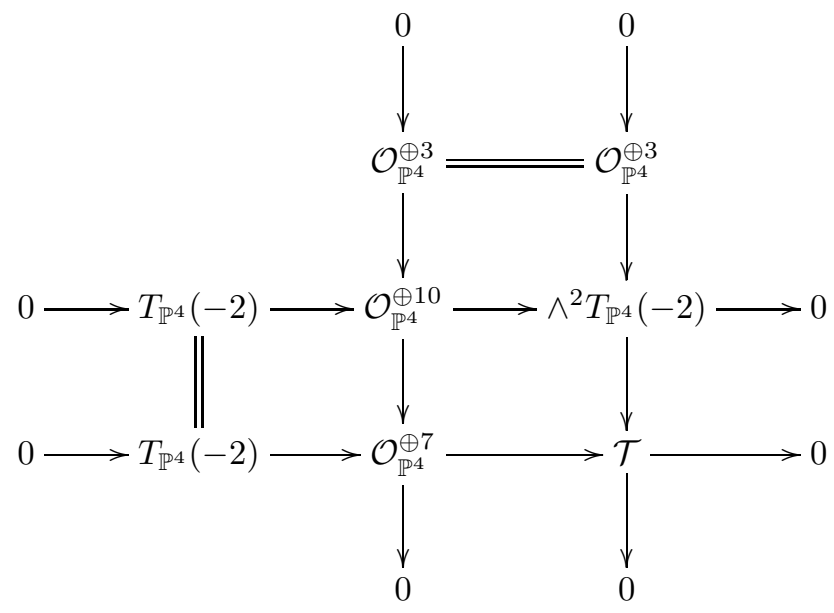


Remark 3. As in [12, one can easily deduce the classification of triple Veronese embeddings of $\mathbb{P}^{r}$ in a Grassmannian of $(k-1)$-planes from Theorem 1.1 and Corollary 1.2. The case $k=2$ has been studied in 8 . Globally generated vector bundles and embeddings in Grassmannians are closely related to matrices of constant rank on projective spaces (see [9] and [7]), but we will not consider this matter in this note.

Remark 4. Following the research initiated in [12, globally generated vector bundles and reflexive sheaves with low first Chern class on projective spaces and quadric hypersurfaces have recently been studied by several authors (see [5], 6], 10], 1], [2], 3] and [4]).

\section{REFERENCES}

[1] C. Anghel, N. Manolache: Globally generated vector bundles on $\mathbb{P}^{n}$ with $c_{1}=3$. arXiv:1202.6261v1 [math.AG]

[2] E. Ballico, S. Huh, F. Malaspina: Globally generated vector bundles of rank 2 on a smooth quadric threefold. arXiv:1211.1100 [math.AG]

[3] E. Ballico, S. Huh, F. Malaspina: On higher rank globally generated vector bundles over a smooth quadric threefold. arXiv:1211.2593 [math.AG]

[4] E. Ballico, S. Huh, F. Malaspina: Reflexive and spanned sheaves on $\mathbb{P}^{3}$. arXiv:1301.1822 [math.AG]

[5] L. Chiodera, Ph. Ellia: Rank two globally generated vector bundles with $c_{1} \leq 5$. Rend. Istit. Mat. Univ. Trieste 44 (2012) 413-422.

[6] Ph. Ellia: Chern classes of rank two globally generated vector bundles on $\mathbb{P}^{2} \cdot \operatorname{arXiv:1111.5718}$ [math.AG]

[7] M.L. Fania, E. Mezzetti: Vector spaces of skew-symmetric matrices of constant rank. Linear Algebra Appl. 434 (2011) 2383-2403.

[8] S. Huh: On triple Veronese embeddings of $\mathbb{P}^{n}$ in the Grassmannians. Math. Nachr. 284 (2011) 1453-1461.

[9] L. Manivel, E. Mezzetti: On linear spaces of skew-symmetric matrices of constant rank. Manuscripta Math. 117 (2005) 319-331.

[10] N. Manolache: Globally generated vector bundles on $\mathbb{P}^{3}$ with $c_{1}=3$. $\operatorname{arXiv:1202.5988} 1$ [math.AG]

[11] Ch. Okonek, M. Schneider, H. Spindler: Vector bundles on complex projective spaces. Progress in Mathematics, 3. Birkhäuser, Boston, Mass., 1980.

[12] J.C. Sierra, L. Ugaglia: On globally generated vector bundles on projective spaces. J. Pure Appl. Algebra 213 (2009) 2141-2146.

Instituto de Ciencias Matemáticas (ICMAT), Consejo Superior de Investigaciones Científicas (CSIC), Campus de Cantoblanco, 28049 Madrid, Spain

E-mail address: jcsierra@icmat.es

Dipartimento di Matematica e Informatica, Università degli Studi di Palermo, Via Archirafi 34, 90123, Palermo, Italy

E-mail address: luca.ugaglia@unipa.it 